\title{
Simultaneous, Dual-Color STORM Imaging at the Cellular Interface
}

\author{
Jesse Aaron*, Bryan Carson*, Jerilyn Timlin* \\ *Dept. Bioenergy and Defense Technologies, Sandia National Laboratories, PO Box 5800, \\ Albuquerque, NM 87185
}

Over the past decade optical approaches have been introduced that effectively break the traditional diffraction barrier. Of particular note were the introductions of Stimulated Emission and Depletion (STED) microscopy [1], Photo-Activated Localization Microscopy (PALM) [2], and the closely related Stochastic Optical Reconstruction Microscopy (STORM) [3]. Of these, STORM represents an attractive method for researchers, as it does not require highly specialized optical setups as is the case in STED, and can be more easily implemented for multi-color imaging.

We implemented a simultaneous dual-color STORM imaging system through the use of an objective-based TIRF microscope (Olympus) and filter-based Optosplit ${ }^{\circledR}$ image splitter (Cairns Research). This system allows for excitation and detection of up to two fluorophors simultaneously, via projection of each fluorophor's signal onto separate regions of the detected. As an initial test of our system, we imaged fluorescent quantum dots (QDs), emitting at 585nm and 655nm, respectively. As has been well characterized, QDs exhibit intermittent "blinking" behavior, allowing for single isolated QDs to be imaged within a diffraction limited area, despite the presence of multiple QDs that are not conventionally resolvable. Separate, simultaneous images of each QD type were acquired using an electron-multiplied CCD (EMCCD, Andor) in concert with the Optosplit ${ }^{\circledR}$. Because of the simultaneous imaging capability, as well as extended sensitivity of the EMCCD detector, a complete two-color data set was acquired in $<10 \mathrm{~s}$. This resulted in the absence of a need for fiduciary markers to correct for stage drift, as has been used in previous studies [3]. After acquisition, each particle's position was identified in postprocessing, and then fit (through a non-linear least-squares algorithm) to a 2D Gaussian function, giving the estimated peak position of the point spread function, with $95 \%$ confidence bounds in the $\pm 10-100 \mathrm{~nm}$ range. Data that resulted in a poor fit $\left(\mathrm{R}^{2}<0.8\right)$ were discarded. Sub-resolution particle positions for each QD type were calculated for each frame, and combined to form a single STORM image, as shown in Figure 1, right, with QD585 indicated in blue and QD655 in orange. For comparison, the same field of view was imaged using conventional TIRF imaging, shown in Figure 1, left. White lines in both images at top indicate areas where pixel intensity profiles were plotted to evaluate the FWHM of the signal peak (Figure 1, bottom). Results indicate a more than 10-fold improvement in image resolution.

We are also utilizing our STORM system to image the sub-resolution organization of the TLR-4 receptor, a key mediator of innate immune response, after challenge with lipopolysaccharide 
(LPS), a bacteria-specific antigen. While distinct forms of LPS have evolved among various bacteria, only some LPS variatoin (such as that derived from $E$. coli) typically result in significant cellular immune response, while others (such as from the plague bacteria $Y$. pestis) do not, despite binding to TLR-4 [4]. We are utilizing our STORM capabilities to reveal potential sub-resolution organizational differences of TLR-4 receptors in the cell membrane in response to binding of various LPS types to elucidate mechanisms of infection

[1] Hell, S.W.; Wichmann, J. (1994), Opt. Lett. 19 (11): 780-782.

[2] Betzig, E., et al. (2006), Science, 313(5793): 1642-1645.

[3] Rust, M.J., et al. (2006), Nat. Meth. 3, 793-796.

[4] James, C.D., et al. (2009) Biomedical Devices 11(3): 693-700.
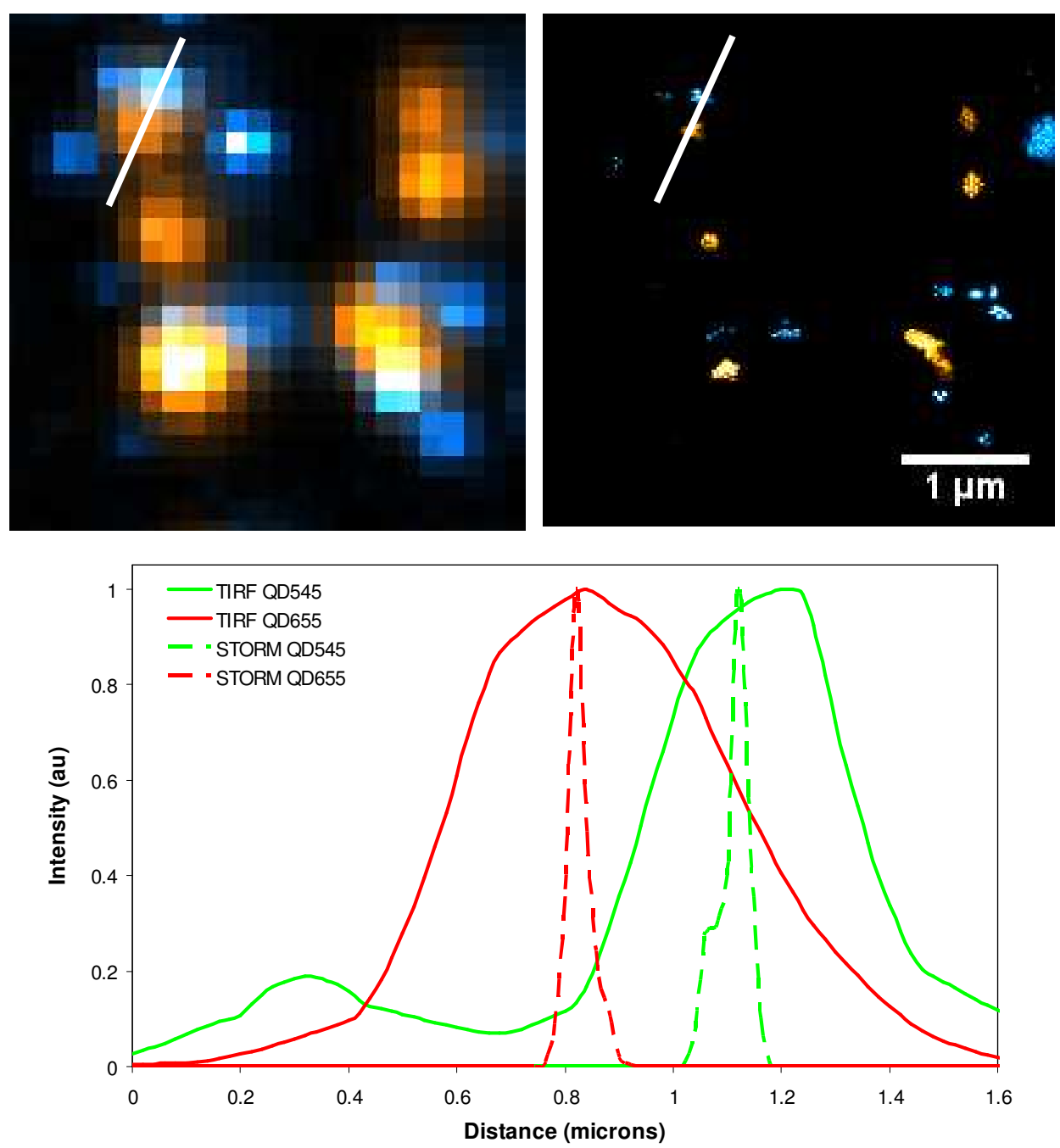

Figure 1. STORM imaging of quantum dots. At top left, a conventional TIRF image of two quantum dot species adhered to a glass substrate (emitting at $585 \mathrm{~nm}$, in blue, and $655 \mathrm{~nm}$, in orange). At top, right, the same field of view is shown following STORM imaging and reconstruction. A complete dual-color data set was acquired in $<10$ seconds. White lines in both images indicate areas where pixel intensity profiles were taken, plotted at bottom. The resolution improvement, as determined by the FWHM of each peak, shows a greater than 10-fold improvement in resolution, to about $40 \mathrm{~nm}$. 